\title{
Pierre Bamony, Des pouvoirs réels du sorcier africain. Forces surnaturelles et autorités sociopolitiques chez les Lyéla du Burkina Faso
}

Paris, L'Harmattan, coll. « Études africaines », 2009, 454 p.

\section{Véronique Duchesne}

\section{(2) OpenEdition Journals}

\section{Édition électronique}

URL : http://journals.openedition.org/assr/24236

DOI : $10.4000 /$ assr.24236

ISSN : $1777-5825$

\section{Éditeur}

Éditions de l'EHESS

Édition imprimée

Date de publication : 30 décembre 2012

Pagination : 117

ISSN : 0335-5985

Référence électronique

Véronique Duchesne, «Pierre Bamony, Des pouvoirs réels du sorcier africain. Forces surnaturelles et autorités sociopolitiques chez les Lyéla du Burkina Faso », Archives de sciences sociales des religions [En ligne], 160 | octobre-décembre 2012, mis en ligne le 21 février 2013, consulté le 21 septembre 2020. URL : http://journals.openedition.org/assr/24236 ; DOI : https://doi.org/10.4000/assr.24236

Ce document a été généré automatiquement le 21 septembre 2020.

(C) Archives de sciences sociales des religions 
Pierre Bamony, Des pouvoirs réels du sorcier africain. Forces surnaturelles et autorités sociopolitiques chez les Lyéla du Burkina Faso

Paris, L'Harmattan, coll. « Études africaines », 2009, 454 p.

Véronique Duchesne

\section{RÉFÉRENCE}

Pierre Bamony, Des pouvoirs réels du sorcier africain. Forces surnaturelles et autorités sociopolitiques chez les Lyéla du Burkina Faso, Paris, L'Harmattan, coll. «Études africaines ", 2009, $454 \mathrm{p}$. 
1 Cet ouvrage est une partie remaniée de la thèse d'anthropologie sociale et d'ethnologie que Pierre Bamony a soutenue en 2001 à l'université BlaisePascal-Clermont II, ce qui explique son caractère volumineux. Dans la première partie est présentée «La région des Lyéla », population autochtone comprenant entre cent mille et cent cinquante mille personnes, qui occupe la province du Sanguié, en plein centreouest du Burkina Faso et qui est restée assez peu étudiée, comparativement aux autres populations du Burkina Faso. L'auteur, lui-même lyélé, explique cela par le fait de l'absence de pouvoir hiérarchisé, à la différence du royaume moose voisin par exemple. Après la présentation du milieu physique et de l'histoire des Lyéla, le chapitre II consacré

\section{Pierre BAMONY}

\section{Des pouvoirs réels du sorcier africain}

Forces sumaturelles et autorités sociopolitiques chez les Lyela du Burkina Faso
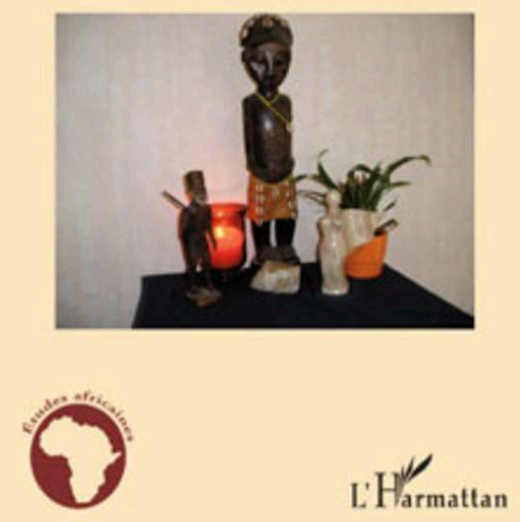
à «La dialectique des pouvoirs dans

l'organisation des villages et communautés » offre une étude stimulante de la gestion de la famille au sein de l'enceinte familiale (kèlè). Les pages sur l'éducation des enfants dans l'art de manger dénotent une compréhension fine des relations familiales, de même que la description des patriclans (kwala). Concernant la bibliographie de cette première partie, on peut noter l'oubli de la thèse de doctorat de Luc Pecquet, Le banco de l'autre. Bâtir les murs d'un ensemble d'habitations en pays Lyela (Burkina Faso) [1999, Université Paris I Panthéon-Sorbonne, 2 vol., 774 p., 20 fig., 79 pl.]. Soulignons par ailleurs que les trois parties qui composent l'ouvrage sont quasi indépendantes et que chacune a sa propre bibliographie, ce qui peut surprendre le lecteur. La deuxième partie, consacrée à la «Conception de la nature, de l'homme et de la religion » revient sur l'idée de l'unicité de Dieu abordée d'un point de vue général chez les peuples subsahariens. Différents mythes portant sur l'origine de phénomènes naturels et sur l'origine de l'homme ont été recueillis directement par l'auteur auprès de traditionalistes aujourd'hui décédés.

2 La thèse que défend Pierre Bamony et qui donne son titre à l'ouvrage est finalement développée, après deux cent vingt-six pages, dans la troisième partie consacrée à la sorcellerie (kialè). L'auteur commence par développer l'idée que la religion est un facteur d'unification politique d'un peuple, à travers l'Ancien Testament, ce qui l'éloigne malheureusement quelque peu de son sujet. Il cherche en effet à démontrer que les personnes obéissent aux lois des patriclans (kwala) par l'intermédiaire de la croyance aux sanctions suprêmes des sorciers (kialè), et explique ainsi l'inexistence « de logistique complexe et coûteuse comme l'armée ou la police, les forces de l'ordre visibles en somme » (p. 234). Son argument aurait gagné à ne pas séparer, comme c'est le cas dans deux chapitres différents, les controverses anthropologiques sur l'essence du pouvoir politique, d'une part, et la notion de pouvoir chez les Lyéla, d'autre part. Les pages relatives au culte de l'autel de la terre sont intéressantes, même si parfois 
quelque peu redondantes. La recension des différents travaux africanistes sur la sorcellerie est tout à fait pertinente, ainsi ayant rappelé que la sorcellerie est un «système » (Augé), l'auteur commence-t-il par décrire la sorcellerie ordinaire avant d'approfondir le système du pouvoir socio-politico-religieux des sorciers, kialè. Il nous montre comment les patriclans lyélé (kwala) ont institué un système d'autorité non visible et comment le pouvoir implicite, toujours invisible mais réel, dense et puissant se fonde sur le collège des kialè (sorciers). La figure de la sorcellerie développée par les Lyéla apparaît ainsi comme le socle de leur équilibre social et politique.

Notons que la mauvaise qualité de l'impression (due à l'éditeur) rend les cartes - au nombre de huit - quasi illisibles. On comprend au fil de la lecture que l'auteur, professeur de philosophie et chercheur anthropologue indépendant, a accumulé un grand nombre de matériaux ethnographiques de première main au cours d'une trentaine d'années (de 1978 à 2006) et qu'il tenait absolument à les faire connaître en les publiant. Ce livre constitue un document précieux pour la connaissance d'une société peu étudiée par ailleurs. Le ton libre, critique et parfois polémique de l'auteur vis-à-vis de la science anthropologique et plus généralement des Européens est appréciable, car il est vrai que " parmi les phénomènes religieux les plus incompris ou les plus abhorrés sont ceux des peuples subsahariens » (p. 175). On peut tout de même regretter à plusieurs reprises les références à des courants de pensée anthropologique tout à fait dépassés, comme l'évolutionnisme du xix ${ }^{e}$ siècle par exemple. Soulignons enfin qu'il a fallu un certain courage à Pierre Bamony pour aborder un sujet aussi délicat que celui de la sorcellerie, quand on est soi-même membre de la société lyélé et plus particulièrement membre d'un patriclan. On pourrait d'ailleurs lui suggérer d'écrire sur les questions méthodologiques qui se sont posées à lui en tant qu'ethnographe sur un terrain qui lui est familier et lui poser cette ultime question: comment ce livre a-t-il été reçu par ses « pères »? 